\title{
Holographic inequalities and entanglement of purification
}

\section{Ning Bao and Illan F. Halpern}

Center for Theoretical Physics and Department of Physics, University of California, Berkeley, CA 94720, U.S.A.

E-mail: ningbao75@gmail.com, illan@berkeley.edu

ABSTRACT: We study the conjectured holographic duality between entanglement of purification and the entanglement wedge cross-section. We generalize both quantities and prove several information theoretic inequalities involving them. These include upper bounds on conditional mutual information and tripartite information, as well as a lower bound for tripartite information. These inequalities are proven both holographically and for general quantum states. In addition, we use the cyclic entropy inequalities to derive a new holographic inequality for the entanglement wedge cross-section, and provide numerical evidence that the corresponding inequality for the entanglement of purification may be true in general. Finally, we use intuition from bit threads to extend the conjecture to holographic duals of suboptimal purifications.

KEYwORDS: AdS-CFT Correspondence, Gauge-gravity correspondence

ARXIV EPRINT: 1710.07643 


\section{Contents}

1 Introduction 1

2 Review of known results 2

2.1 Basic properties of $E_{W}$ and $E_{p} \quad 2$

2.2 Entanglement entropy inequalities 3

3 Bounding holographic entanglement entropy with $E_{W} \quad 4$

3.1 Generalized $E_{W} \quad 4$

$3.2 E_{W}^{G}$ obeys known $E_{W}$ inequalities 4

3.3 Upper bounding holographic conditional mutual information 5

$\begin{array}{ll}3.4 & \text { Upper bounding holographic tripartite information }\end{array}$

$\begin{array}{lll}3.5 & \text { Upper bounding holographic cyclic information } & 7\end{array}$

$\begin{array}{lll}3.6 & \text { Cyclic } E_{W} \text { inequalities } & 8\end{array}$

4 Bounding entanglement entropy with $E_{p} \quad 8$

4.1 Generalized $E_{p} \quad 8$

$4.2 E_{p}^{G}$ obeys known $E_{p}$ inequalities 9

4.3 Upper bounding conditional mutual information 9

4.4 Upper bounding tripartite information and cyclic information $\quad 10$

$\begin{array}{lll}4.5 & \text { Lower bound on tripartite information } & 10\end{array}$

$\begin{array}{lll}4.6 & \text { Cyclic } E_{p} \text { inequalities } & 10\end{array}$

5 Future direction: new dictionary entries $\quad 12$

6 Conclusion 13

\section{Introduction}

There has been much recent interest in the interplay between the fields of quantum information and quantum gravity. One central point of interest is on the discussions of notions of entanglement measures in the context of the AdS/CFT correspondence [1,2]. In particular, the holographic formula relating entanglement entropy to bulk area of a boundary homologous minimal surface by Ryu and Takayanagi [3, 4] (later extended to extremal surfaces in the covariant case by [5]) has spurred a great deal of interest, from its ability to constrain what sets of states can be dual to classical bulk gravity theories $[6,7]$ to its role as motivation for the idea that the gravity theory is emergent from the entanglement properties of the boundary field theory [8-11].

Even more recently work has been done $[12,13]$ conjecturing that a holographic object, the entanglement wedge cross section $E_{W}$ separating two regions, is dual to the information 
theoretic concept of the entanglement of purification $E_{p}$. This conjecture, which we refer to as the $E_{W}=E_{p}$ conjecture, was made on the basis that $E_{W}$, a holographic object, obeys the same set of inequalities that $E_{p}$ is known to obey. This would be a compelling correspondence, as it is not known how to calculate $E_{p}$ for generic quantum states, whereas $E_{W}$ is an often finite geometric quantity that is simply calculable.

In this work, we will study and generalize the relationship between $E_{W}, E_{p}$, and the holographic entanglement entropy inequalities in three ways: first, we investigate whether $E_{W}$ can nontrivially bound combinations of entanglement entropies that appear in the holographic entropy inequalities; second, we check whether $E_{p}$ provably provides the same type of bounds to these objects; lastly, we ask whether one can extend the $E_{W}=E_{p}$ conjecture to suboptimal purifications and cuts of the entanglement wedge. We will find that the answers to all three of these questions appear to be affirmative, thus providing more evidence for the $E_{W}=E_{p}$ conjecture of $[12,13]$.

\section{Review of known results}

\subsection{Basic properties of $E_{W}$ and $E_{p}$}

Let us define both the entanglement wedge cross section $E_{W}$, and the entanglement of purification $E_{p}$. First, we define holographic states to be quantum states of the boundary conformal field theory that are dual to a well defined classical bulk gravitational theory in AdS/CFT. For a holographic state, the entanglement wedge cross-section is defined for any two regions of time reversal symmetric slices (though the generalization to the fully covariant case exists in [13]) as

$$
E_{W}(A: B)=\min \left\{\operatorname{Area}(\Gamma) ; \Gamma \subset r_{A B} \text { splits } A \text { and } B\right\}
$$

where $r_{A B}$ is the entanglement wedge ${ }^{1}[14]$ of $A B=A \cup B$ (see figure 1). In words, $E_{W}$ is the minimal area of a surface $\Gamma$ that splits $r_{A B}$ into two regions, one of which is bounded by $A$ but not $B$, and other by $B$ but not $A$. If $s_{A}, s_{B}$ and $s_{A B}$ denote Ryu-Takayanagi (RT) surfaces, then we want $\Gamma$ to split $r_{A B}=r_{A B}^{(A)} \sqcup r_{A B}^{(B)}$ (here $\sqcup$ denotes disjoint union) and $s_{A B}=s_{A B}^{(A)} \sqcup s_{A B}^{(B)}$ with $\partial r_{A B}^{(A)}=A \cup s_{A B}^{(A)} \cup \Gamma$. $^{2}$ In this work, we refer interchangeably to the surface and the area thereof as the entanglement wedge cross-section, but the meaning should be clear from context.

Now, consider an arbitrary bipartite quantum system $A B$. The entanglement of purification $E_{p}(A: B)$ is defined by

$$
E_{p}(A: B)=\min \left\{S\left(A A^{\prime}\right) ; A A^{\prime} B B^{\prime} \text { pure }\right\}
$$

where $S$ is the Von Neumann entropy (see figure 1). Note that because the overall state is pure this is symmetric under $A \leftrightarrow B$.

\footnotetext{
${ }^{1}$ Technically, $r_{A B}$ is the restriction of the entanglement wedge to the time symmetric slice under consideration. Since we will only be concerned with this time symmetric situation, and all objects considered live on this slice, we leave this restriction implicit throughout.

${ }^{2}$ It is also worth noting that $E_{W}$ (and its to-be-developed generalization) is finite if the regions being split are nonadjacent in the boundary theory.
} 

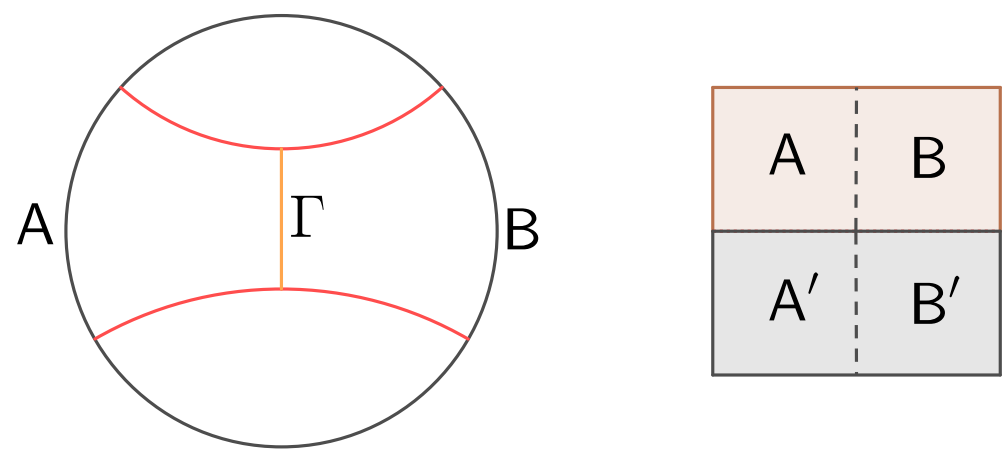

Figure 1. To the left, $\Gamma$ is the minimal surface the separates the entanglement wedge cross-section of $A B$. Its area is $E_{W}[A: B]$. To the right, $A^{\prime}$ and $B^{\prime}$ purify $A B$. For a choice of $A^{\prime}$ and $B^{\prime}$ over all such purifying systems that minimizes the entanglement across the dashed partition we have $E_{p}(A: B)=S\left(A A^{\prime}\right)$.

Both $E_{W}$ and $E_{p}$ are known to satisfy the following inequalities:

$$
\begin{aligned}
\min \left(S_{A}, S_{B}\right) & \geq E(A: B) \geq \frac{1}{2} I(A: B) \\
E(A: B C) & \geq E(A: B) \\
E(A B: C) & \geq \frac{1}{2}(I(A: C)+I(B: C)),
\end{aligned}
$$

where $E$ here can stand for either $E_{p}$ or $E_{W}$, and $I(A: B) \equiv S(A)+S(B)-S(A B)$ is the mutual information between $A$ and $B$. We refer the reader to [13] for clear proofs of these inequalities in the context of $E_{W}$, and $[15,16]$ for the same for $E_{p}$. These coinciding bounds for $E_{p}$ and $E_{W}$ is what motivated the conjecture of $[12,13]$ that $E_{W}$ is the holographic dual of $E_{p}$.

\subsection{Entanglement entropy inequalities}

Before we study potential new inequalities for $E_{p}$ and $E_{W}$, let's list some known inequalities for entanglement entropy that will prove useful in the upcoming discussion. For holographic proofs of these inequalities we refer the reader to $[6,7,17]$. All tripartite quantum states satisfy strong subadditivity (SSA):

$$
I(A: B \mid C) \equiv S(B C)+S(A C)-S(A B C)-S(C) \geq 0,
$$

where $I(A: B \mid C)$ is the conditional mutual information. When $C=\emptyset$, this reduces to subadditivity, or positivity of the mutual information. Moreover, all holographic states satisfy monogamy of mutual information (MMI) [7]:

$$
\begin{aligned}
& I(A: B C) \geq I(A: B)+I(A: C) \\
& \quad \Leftrightarrow I(A: B: C) \equiv I(A: B C)-I(A: B)-I(A: C) \geq 0,
\end{aligned}
$$

where $I(A: B: C)$ is the tripartite information, which is symmetric under permutations of its arguments. 
It is worth stressing that not all quantum states satisfy MMI. For example, the GHZ state defined by $|\mathrm{GHZ}\rangle=\frac{1}{\sqrt{2}}\left(|0\rangle^{\otimes n}+|1\rangle^{\otimes n}\right)$ does not do so for $n \geq 4$.

Recently, several further holographic entanglement entropy inequalities were proven [6]. Among them, there's an infinite family of cyclic inequalities given by

$$
C_{k}\left(A_{1}, \ldots, A_{n}\right) \equiv \sum_{i=1}^{n} S\left(A_{i} \mid A_{i+1} \ldots A_{i+k}\right)-S\left(A_{1} \ldots A_{n}\right) \geq 0,
$$

where $n=2 k+1$, the indices are interpreted $\bmod n, S(A \mid B)=S(A B)-S(B)$ is the conditional entropy, and $C_{k}$ is what we call the $k$-cyclic information (or just cyclic information). For $k=1$, eq. (2.8) gives MMI, but for $k>1$ it gives a family of new and independent inequalities.

\section{Bounding holographic entanglement entropy with $E_{W}$}

\subsection{Generalized $E_{W}$}

In order to bound holographic entanglement entropy with $E_{W}$, we first slightly generalize the notion of entanglement wedge cross-section $E_{W}(A: B)$ to allow for $A \cap B \neq \emptyset$. A generalization of $E_{p}(A: B)$ for this case will be given in section 4.1.

The generalized entanglement wedge cross-section $E_{W}^{G}$ is defined as

$$
E_{W}^{G}(A: B)=\min \left\{\operatorname{Area}(\Gamma) ; \Gamma \subset r_{A B}-r_{A \cap B} \text { splits } A \backslash B \text { and } B \backslash A\right\},
$$

so now the surface $\Gamma$ separates $A \backslash B$ from $B \backslash A$ in the region defined by the entanglement wedge of $A B$ with the entanglement wedge of $A \cap B$ removed. ${ }^{3}$ Note that if $A \cap B=\emptyset$ then $E_{W}^{G}(A: B)=E_{W}(A: B)$.

We also define a convenient form of mutual information, $I^{G}(A: B)=I(A \backslash B, B \backslash A)=$ $S(A \backslash B)+S(A \backslash B)-S(A \backslash B \cup B \backslash A)$. Similiarly, if the intersection between $A$ and $B$ is trivial this reduces to $I(A: B)$.

\section{$3.2 \quad E_{W}^{G}$ obeys known $E_{W}$ inequalities}

In this section, we show that this generalized entanglement wedge cross-section obeys the known inequalities for $E_{W}$ in eqs. (2.3)-(2.5). In section 4.2 we will show that a suitably generalized $E_{p}$ also obeys these inequalities. Thus there is as much evidence for the generalized conjecture $E_{W}^{G}=E_{p}^{G}$ as there is for the original $E_{W}=E_{p}$ conjecture.

The upper bound in eq. (2.3) follows from

$$
\begin{aligned}
& E_{W}^{G}(A: B) \leq E_{W}(A: B \backslash A) \leq \min (S(A), S(B \backslash A)) \quad \text { and } \\
& E_{W}^{G}(A: B) \leq E_{W}(A \backslash B: B) \leq \min (S(A \backslash B), S(B)),
\end{aligned}
$$

where the first inequalities above follow from the fact that for $E_{W}^{G}(A: B)$ is the minimum area curve $\Gamma$ that separates $A \backslash B$ from $B \backslash A$ in $r_{A B}-r_{A \cap B}$, and so it can be no longer than optimal curve separating these same regions in $r_{A B}$.

\footnotetext{
${ }^{3}$ For an illustration of what this generalization means geometrically, see figure 2 , in which $E_{W}^{G}(A C$ : $B C)=\operatorname{Area}(\Gamma))$.
} 
The lower bound follows from $r_{A B \backslash(A \cap B)} \subset\left(r_{A B}-r_{A \cap B}\right)$, which is a consequence of entanglement wedge nesting $(\mathrm{EWN})[18,19]$ and implies

$$
E_{W}^{G}(A: B) \geq E_{W}(A \backslash B: B \backslash A) \geq \frac{1}{2} I^{G}(A: B) .
$$

It follows from entanglement wedge nesting that if $A \cap C=\emptyset$, then

$$
E_{W}^{G}(A: B C) \geq E_{W}^{G}(A: B) .
$$

Finally,

$$
E_{W}^{G}(A: B C) \geq \frac{1}{2}\left(I^{G}(A: B)+I(A \backslash B: C)\right)
$$

follows from eq. (3.3), MMI, and the disjointedness of $A$ and $C$.

\subsection{Upper bounding holographic conditional mutual information}

One can ask the question of whether or not holography in general, and $E_{W}^{G}$ in particular, provides an upper bound to the conditional mutual information. We note that this question was first answered in the affirmative by [20] using bit threads, but it is instructive to treat it again here.

The holographic bound for $I(A: B \mid C)$ in [20] reads:

$$
I(A: B \mid C) \leq 2 E_{W}^{G}(A C: B C) .
$$

Note that in the case where $C=\emptyset$, this reduces to $I(A: B) \leq 2 E_{W}(A: B)$.

This upper bound can also be proven using exclusion/inclusion [17] or equivalently graph contraction [6], with the main new technique used being that the cutting and regluing procedure is no longer constrained to only boundary anchored minimal surfaces, but potentially includes bulk-anchored minimal surfaces such as the entanglement wedge crosssection as well. See figure 2 .

Let's now follow [17] in putting into equations what figure 2 shows. Let $s_{X}$ denote the RT surface of some boundary region $X$, and $r_{X}$ denote its entanglement wedge so that the boundary of $r_{X}$ is $\partial r_{X}=X \cup s_{X}$.

Let $A, B$, and $C$ be disjoint regions, and let $R=r_{A B C} \backslash r_{C}$. By EWN, $\partial R=s_{a b c} \cup s_{c}$. Let $\Gamma$ be the surface that satisfies the minimization in $E_{W}[A C: B C]$. Then, it splits $R$ into two disjoint regions $R^{(A)}$ and $R^{(B)}$ such that

$$
\begin{aligned}
& \partial R^{(A)}=A+\Gamma+s_{A B C}^{(A)}+s_{C}^{(A)} \\
& \partial R^{(B)}=B+\Gamma+s_{A B C}^{(B)}+s_{C}^{(B)},
\end{aligned}
$$

where $s_{C}=s_{C}^{(A)} \sqcup s_{C}^{B}$ and $s_{A B C}=s_{A B C}^{(A)} \sqcup s_{A B C}^{B}$. Then,

$$
\begin{aligned}
& \partial\left(r_{C} \cup R^{(A)}\right)=(A \cup C) \cup\left(\Gamma \cup s_{C}^{(B)} \cup s_{A B C}^{(A)}\right) \\
& \partial\left(r_{C} \cup R^{(B)}\right)=(A \cup B) \cup\left(\Gamma \cup s_{C}^{(A)} \cup s_{A B C}^{(B)}\right)
\end{aligned}
$$



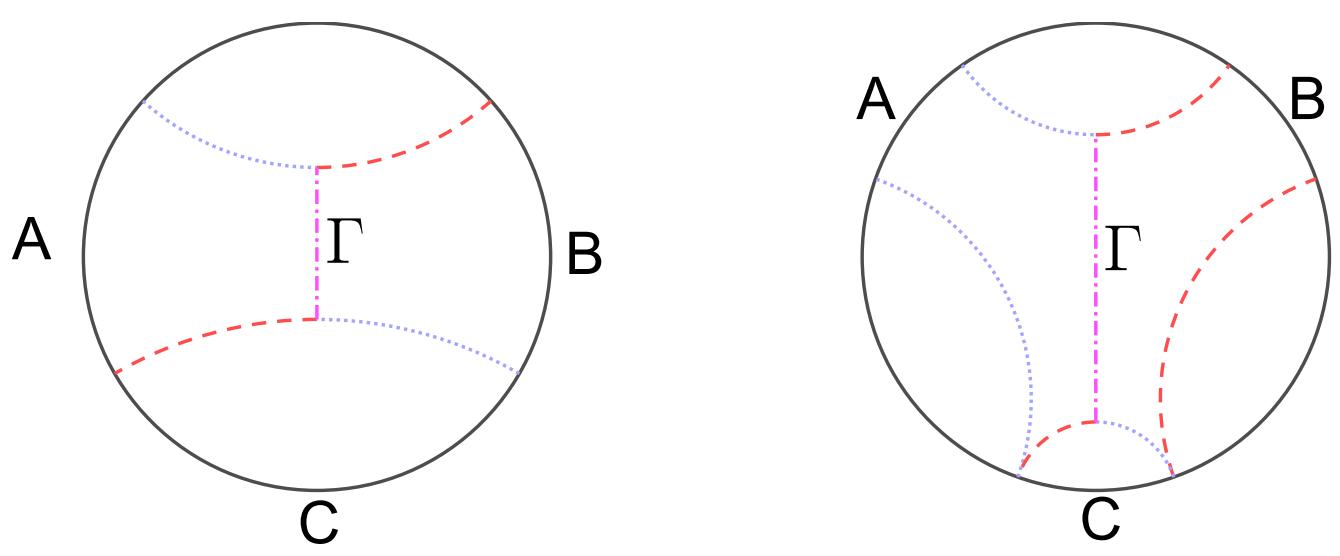

Figure 2. Graphical proof of the upper bound on the conditional mutual information for both the case in which the regions $\mathrm{A}, \mathrm{B}$, and $\mathrm{C}$ are contiguous and the case in which they are disconnected. It is clear from the diagrams,and Ryu-Takayanagi, that the area of the dotted surfaces plus the area of the dash-dotted surface is greater than or equal $S(A C)$ and that the area of the dashed surfaces plus the area of the dash-dotted surface is greater than or equal $S(B C)$. Adding these two inequalities gives us the desired bound.

Then, by RT, eq. (3.8) implies that the area of $\left(\Gamma \cup s_{C}^{(B)} \cup s_{A B C}^{(A)}\right)$ is greater than or equal to $S_{A C}$, and eq. (3.8) implies that the area of $\left(\Gamma \cup s_{C}^{(A)} \cup s_{A B C}^{(B)}\right)$ is greater than or equal to $S_{B C}$. Adding these two inequality, and applying RT again, we get the desired inequality

$$
2 E_{W}[A C: B C] \geq S_{A C}+S_{B C}-S_{A B C}-S_{C}=I(A: B \mid C) \geq 0
$$

where we used the positivity of the conditional mutual information.

\subsection{Upper bounding holographic tripartite information}

We can also use $E_{W}^{G}$ to upper bound holographic tripartite information:

$$
I(A: B: C) \leq E_{W}^{G}(A C: B C)+E_{W}^{G}(A B: B C)+E_{W}^{G}(C B: A C) .
$$

We could have pursued an inclusion-exclusion style proof for this, but amusingly one does not have to; this follows from eq. (3.6). Adding three instances of eq. (3.6), we get:

$$
\begin{aligned}
E_{W}^{G}(A C & : B C)+E_{W}^{G}(A B: C B)+E_{W}^{G}(B A: C A) \\
& \geq \frac{1}{2}(I(A: B \mid C)+I(A: C \mid B)+I(B: C \mid A)) \\
& =S_{A B}+S_{A C}+S_{B C}-\frac{3}{2} S_{A B C}-\frac{1}{2}\left(S_{A}+S_{B}+S_{C}\right) \\
& \geq S_{A B}+S_{A C}+S_{B C}-S_{A B C}-S_{A}-S_{B}-S_{C}
\end{aligned}
$$

where in the last line, we used three party subadditivity $\left(S_{A B C} \leq S_{A}+S_{B}+S_{C}\right)$. We recognize the last expression above as $I(A: B: C)$, thus completing the proof. 


\subsection{Upper bounding holographic cyclic information}

Similarly, the following upper bound on $C_{k}$ can also be derived:

$$
\sum_{i=1}^{n} E_{W}^{G}\left(A_{i}, A_{i+i}, \ldots, A_{i+k}: A_{i+k}, A_{i+k+1}, \ldots A_{i+n-1}\right) \geq C_{k}\left(A_{1}, \ldots, A_{n}\right),
$$

where, as before, the indices are to be interpreted $\bmod n$, and $n=2 k+1$.

To prove this, we use eq. (3.6) to get:

$$
\begin{aligned}
\sum_{i=1}^{n} E_{W}^{G}( & \left.A_{i}, \ldots, A_{i+k}: A_{i+k}, \ldots A_{i+n-1}\right) \\
& \geq \sum_{\text {cус }} S\left(A_{1} \cdots A_{k+1}\right)-\frac{n}{2} S\left(A_{1} A_{2} \ldots A_{n}\right)-\frac{1}{2} \sum_{j=1}^{n} S\left(A_{j}\right) \\
& \geq \sum_{\text {сус }} S\left(A_{1} \cdots A_{k+1}\right)-S\left(A_{1} A_{2} \ldots A_{n}\right)-\frac{n-2}{2} S\left(A_{1} A_{2} \ldots A_{n}\right)-\frac{1}{2} \sum_{j=1}^{n} S\left(A_{j}\right) \\
& \geq C_{k}\left(A_{1} \ldots A_{n}\right),
\end{aligned}
$$

where we have used subadditivity and that

$$
2 \sum_{\text {cyclic }} S\left(A_{1} \ldots A_{k}\right) \geq(n-2) S\left(A_{1} \ldots A_{n}\right)+\sum_{j} S\left(A_{j}\right),
$$

which follows from repeated application of SSA, as we now show. First, pairwise application of SSA to terms of the form $S\left(A_{i}, \ldots, A_{k}\right)$ and $S\left(A_{k}, \ldots, A_{2 k-1}\right)$ on the left-hand side gives:

$$
2 \sum_{\text {cyclic }} S\left(A_{1} \ldots A_{k}\right) \geq \sum_{j} S\left(A_{j}\right)+\sum_{\text {cyc }} S\left(A_{1} \ldots A_{2 k-1}\right)
$$

Now, let $F$ be a purification of $A_{1} \ldots A_{n}$, so that we have $\sum_{\text {cyc }} S\left(A_{1} \ldots A_{2 k-1}\right)=$ $\sum_{i} S\left(A_{i} A_{i+1} F\right)$. Applying SSA now to $S\left(A_{2 i} A_{2 i+1} F\right)$ for $i=1, \ldots, k$, and to $S\left(A_{2 i-1} A_{2 i} F\right)$ for $i=1, \ldots k$ we get

$$
\begin{aligned}
\sum_{i} S\left(A_{i} A_{i+1} F\right) & \geq(n-1) S(F)+S\left(A_{1} A_{n} F\right)+S\left(A_{1} \ldots A_{n-1} F\right)+S\left(A_{2} \ldots A_{n} F\right) \\
& \geq(n-2) S(F)=(n-2) S\left(A_{1} \ldots A_{n}\right) .
\end{aligned}
$$

Finally, pairwise application of SSA to $S\left(A_{i} \ldots A_{i+k+1}\right)$ and $S\left(A_{i+k+1} \ldots A_{i+n-1}\right)$ for $i=1$ to $k$ gives

$$
\begin{aligned}
\sum_{\text {сус }} S\left(A_{1} \ldots A_{k+1}\right) & \geq k S\left(A_{1} \ldots A_{n}\right)+\sum_{i=1}^{k} S_{i}+S\left(A_{k+1} A_{k+2} \ldots A_{n}\right) \\
& \geq(k+1) S\left(A_{1} \ldots A_{n}\right) .
\end{aligned}
$$

Combining eqs. (3.16), (3.17), and (3.18) yields eq. (3.15). 


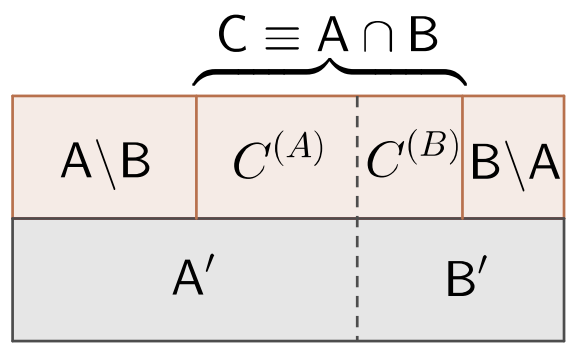

Figure 3. As depicted, for the optimal choices of $A^{\prime}, B^{\prime}$ and $C^{(A)}$, we have $E_{p}^{G}(A: B)=$ $S\left((A \backslash B) A^{\prime} C^{(A)}\right)$.

\subsection{Cyclic $E_{W}$ inequalities}

Here we use as a starting point the cyclic entropy inequalities, eq. (2.8), to derive cyclic $E_{W}$ inequalities. Interestingly, as we show in section 4 , the inequalities we arrive are not obviously violated for generic quantum states when $E_{W}$ is replaced by $E_{p}$.

We first rewrite the cyclic entropy cone inequalities, eq. (2.8), in terms of only mutual information as

$$
\sum_{i=2}^{n} I\left(A_{1} A_{2} \ldots A_{i-1}: A_{i}\right) \geq \sum_{\text {cyclic }} I\left(A_{1}: A_{2} \ldots A_{1+k}\right) .
$$

Then, by the upper bound in eq. (2.3), the left-hand side of the inequality above can be upper bounded by a combination of $E_{W}$ 's, which gives

$$
\sum_{i=2}^{n} E_{W}\left(A_{1} A_{2} \ldots A_{i-1}: A_{i}\right) \geq \frac{1}{2} \sum_{\text {cyclic }} I\left(A_{1}: A_{2} \ldots A_{1+k}\right) .
$$

\section{Bounding entanglement entropy with $E_{p}$}

\subsection{Generalized $E_{p}$}

Just as we did for $E_{W}$, we will similarly need to generalize $E_{p}$. The generalized entanglement of purification $E_{p}^{G}$ is defined as $^{4}$

$$
E_{p}^{G}(A: B)=\min _{A^{\prime} B^{\prime} C^{(A)}} S\left((A \backslash B) A^{\prime} C^{(A)}\right),
$$

where as before we require that $A A^{\prime} B B^{\prime}$ is pure, and we now also require that $C^{(A)} \subset$ $C \equiv A \cap B$. For convenience, we also define $C^{(B)}=C \backslash C^{(A)}$ (see figure 3). Note that the minimization could also have been done over $S\left((B \backslash A) B^{\prime} C^{(B)}\right)$ and also that if $A \cap B=\emptyset$ then $E_{p}^{G}(A: B)=E_{p}(A: B)$. Moreover, even when $A, B$ and $C$ have a geometrical interpretation (as is the case when they have a holographic bulk dual), there is no requirement that the split of $C$ into $C^{(A)}$ and $C^{(B)}$ be geometric.

\footnotetext{
${ }^{4} \mathrm{~A}$ different generalization was proposed in [21].
} 


\section{2 $E_{p}^{G}$ obeys known $E_{p}$ inequalities}

We now show that $E_{p}^{G}$ also obeys the known inequalities for $E_{p}$.

The upper bound in eq. (2.3) follows from

$$
\begin{aligned}
& E_{p}^{G}(A: B) \leq E_{p}(A: B \backslash A) \leq \min (S(A), S(B \backslash A)) \quad \text { and } \\
& E_{p}^{G}(A: B) \leq E_{p}(A \backslash B: B) \leq \min (S(A \backslash B), S(B)),
\end{aligned}
$$

where the first inequality in each line follows from the fact the minimization procedure defining $E_{p}^{G}(A: B)$ is less constrained than the one defining $E_{p}(A: B \backslash A)$ or $E_{p}(A \backslash B: B){ }^{5}$ and the second inequality in each line follows from eq. (2.3). Together these imply

$$
E_{p}^{G}(A: B) \leq \min (S(A), S(B)) .
$$

The lower bound in eq. (2.3) follows from

$$
\begin{aligned}
E_{p}^{G}(A: B) & \geq E_{p}(A \backslash B: B \backslash A) \\
& \geq \frac{1}{2}(S(A \backslash B)+S(B \backslash A)-S(A \backslash B \cup B \backslash A))=I^{G}(A: B),
\end{aligned}
$$

where we have used the fact that the minimization procedure for $E_{p}^{G}(A: B)$ is more constrained that the one for $E_{p}(A \backslash B: B \backslash A),{ }^{6}$ and eq. (2.3).

For any $C \cap A=\emptyset$, it is easy to see that

$$
\begin{aligned}
& E_{p}^{G}(A: B C) \geq E_{p}(A: B), \quad \text { and } \\
& E_{p}^{G}(A: B C) \geq E_{P}(A-B,(B-A) C) \geq \frac{1}{2}\left(I^{G}(A: B)+I(A \backslash B: C)\right)
\end{aligned}
$$

since adding $C$ further constrains the optimization.

\subsection{Upper bounding conditional mutual information}

We now prove the following upper on conditional mutual information:

$$
I(A: B \mid C) \leq 2 E_{p}^{G}(A C: B C) .
$$

Note that, similarly to the $E_{W}^{G}$ bound, in the case where $C=\emptyset$, this reduces to $I(A: B) \leq$ $2 E_{p}(A: B)$.

Assume $A \cap B=\emptyset$, and let $A^{\prime}, B^{\prime}$, and $C^{(A)} \subset C$ define an optimal purification of $(A C: B C)$ according to eq. (4.1). Then, we can get the desired upper bound by repeated application of strong subadditivity:

$$
\begin{aligned}
2 E_{p}^{G}(A C & : B C)+S(A B C)+S(C) \\
& =S\left(A A^{\prime} C^{(A)}\right)+S\left(B B^{\prime} C^{(B)}\right)+S(A B C)+S(C) \\
& \geq S\left(A A^{\prime} B C\right)+S\left(A C^{(A)}\right)+S\left(B B^{\prime} C^{(B)}\right)+S\left(C^{(A)} C^{(B)}\right) \\
& \geq S\left(A A^{\prime} B C\right)+S\left(B B^{\prime} C^{(B)}\right)+S\left(C^{(A)}\right)+S(A C) \\
& \geq S\left(B C^{(B)}\right)+S(A C)+S\left(C^{(A)}\right) \geq S(B C)+S(A C) .
\end{aligned}
$$

\footnotetext{
${ }^{5}$ This is because for $E_{p}^{G}(A: B)$ we are free to choose $C^{(A)}$, while in $E_{p}(A: B \backslash A)$ we have that $C^{(A)}=\emptyset$, and in $E_{p}(A \backslash B: B)$ we have $C^{(A)}=C=A \cap B$.

${ }^{6}$ This follows from the fact that we can always take $C^{(A)}$ to be part of $A^{\prime}$ and $C^{(B)}$ part of $B^{\prime}$.
} 


\subsection{Upper bounding tripartite information and cyclic information}

It is worth noting that the proofs in the previous section of upper bounds for holographic tripartite information, eq. (3.11), and holographic cyclic information, eq. (3.13), depended only on eq. (3.6). Since the analogous statement obtained by replacing $E_{W}^{G}$ by $E_{p}^{G}$, i.e., eq. (4.7), also holds, the $E_{p}^{G}$ versions of these upper bounds are also true.

\subsection{Lower bound on tripartite information}

One can also extract a quantum lower bound for the tripartite information:

$$
I(A: B: C) \geq-2 E_{p}(A: B C)-2 E_{p}(B: C) .
$$

This inequality is obviated in the holographic context by positivity of holographic tripartite information. For a general quantum state, however, it is nontrivial. To prove this inequality, we add three instances of positivity of conditional mutual information to find:

$$
\begin{aligned}
I(A: B \mid C) & +I(A: C \mid B)+I(B: C \mid A) \\
& =2 S(A B)+2 S(B C)+2 S(A C)-S(A)-S(B)-S(C)-3 S(A B C) \geq 0 .
\end{aligned}
$$

We can add to this inequality the inequality

$$
S(A B C)-S(A)-S(B)-S(C) \geq-2 E_{p}(A: B C)-2 E_{p}(B: C),
$$

which follows from two applications of eq. (2.3). The sum of eqs. (4.10) and (4.11) proves the lower bound in eq. (4.9).

\subsection{Cyclic $E_{p}$ inequalities}

If the $E_{W}=E_{p}$ conjecture is correct, then for holographic states, it follows from eq. (3.20) that

$$
\sum_{i=2}^{n} E_{p}\left(A_{1} A_{2} \ldots A_{i-1}: A_{i}\right) \geq \frac{1}{2} \sum_{\text {cyclic }} I\left(A_{1}: A_{2} \ldots A_{1+k}\right)
$$

However, it is interesting to note that in deriving this, we have combined several inequalities, thereby weakening them. For instance, the GHZ state defined by $|\mathrm{GHZ}\rangle=$ $\frac{1}{\sqrt{2}}\left(|0\rangle^{\otimes n}+|1\rangle^{\otimes n}\right)$ is not holographic for $n \geq 4$ and violates instances of eq. (2.8), but still satisfies eq. (3.20). This can be seen from the fact that for any $A$ and $B$ disjoint proper subsystems of GHZ, we have [16]:

$$
E_{p}(A: B)=S(A)=S(B) \text {, and } I(A: B)=S(A)=S(B) .
$$

Thus all the terms in Eq. (3.19) and eq. (4.12) are the same, and we can see the former is violated, while the latter satisfied.

Thus, it is plausible that the inequalities in eq. (4.12) hold for general quantum states. Because random states are known to obey the holographic inequalities [22], it is also clear that those states would also obey eq. (4.12). 
We now present evidence that these inequalities are also obeyed by $\mathrm{W}$ states, which are also known not to be holographic and are defined by

$$
|W\rangle=\frac{1}{\sqrt{n}}(|100 \ldots 0\rangle+|010 \ldots 0\rangle+\cdots|00 \ldots 01\rangle) .
$$

For any qubit system invariant under the permutation of qubits, we have

$$
\sum_{\text {cyclic }} I\left(A_{1}: A_{2} \ldots A_{1+k}\right)=n\left(S\left(\rho_{1}\right)+S\left(\rho_{k}\right)-S\left(\rho_{k+1}\right)\right),
$$

where $\rho_{i}$ is the reduced density matrix for the i-qubit subsystem. Moreover, by using eq. (2.5) and permutation symmetry, we can lower bound the left-hand side of eq. (4.12) as follows:

$$
\sum_{i=2}^{n} E_{p}\left(A_{1} A_{2} \ldots A_{i-1}: A_{i}\right) \geq \frac{1}{2}\left(6 k S\left(\rho_{1}\right)-2 k S\left(\rho_{2}\right)-S\left(\rho_{2 k}\right)\right)
$$

Thus, eq. (4.12) is implied by

$$
D \equiv(4 k-1) S\left(\rho_{1}\right)-2 k\left(S\left(\rho_{2}\right)\right)-S\left(\rho_{2 k}\right)-(2 k+1) S\left(\rho_{k}\right)(2 k+1)+S\left(\rho_{k+1}\right) \geq 0 .
$$

Let $W_{n}$ be the density matrix for the $n$ qubit $\mathrm{W}$ state, and let $W_{n, k}$ be its reduced density matrix to a $k$ qubit subsystem. We can now write these in component form as

$$
W_{n}=\frac{1}{n}\left(\begin{array}{cccccccc}
0 & 0 & 0 & 0 & 0 & 0 & \cdots & 0 \\
0 & 1 & 1 & 0 & 1 & 0 & 0 & 0 \\
0 & 1 & 1 & 0 & 1 & 0 & 0 & 0 \\
0 & 0 & 0 & 0 & 0 & 0 & 0 & 0 \\
0 & 1 & 1 & 0 & 1 & 0 & 0 & 0 \\
0 & 0 & 0 & 0 & 0 & 0 & 0 & 0 \\
\vdots & 0 & 0 & 0 & 0 & 0 & \ddots & 0 \\
0 & 0 & 0 & 0 & 0 & 0 & 0 & 0
\end{array}\right),
$$

where row $i$ column $j$ contains a 1 if and only if $i-1$ and $j-1$ are powers of 2 , and

$$
W_{n, k}=\frac{1}{n}\left(\begin{array}{cccccccc}
n-k & 0 & 0 & 0 & 0 & 0 & \cdots & 0 \\
0 & 1 & 1 & 0 & 1 & 0 & 0 & 0 \\
0 & 1 & 1 & 0 & 1 & 0 & 0 & 0 \\
0 & 0 & 0 & 0 & 0 & 0 & 0 & 0 \\
0 & 1 & 1 & 0 & 1 & 0 & 0 & 0 \\
0 & 0 & 0 & 0 & 0 & 0 & 0 & 0 \\
\vdots & 0 & 0 & 0 & 0 & 0 & \ddots & 0 \\
0 & 0 & 0 & 0 & 0 & 0 & 0 & 0
\end{array}\right),
$$

where, apart from the first entry, the same pattern is followed. This allows us to evaluate the left-hand side of eq. (4.17) and verify its positivity (for numerically tractible $n$ and $k$ ). Moreover, the best fit we found for these curves indicate that this is satisfied for any value of $k$ and $n$ (See figure 4). 


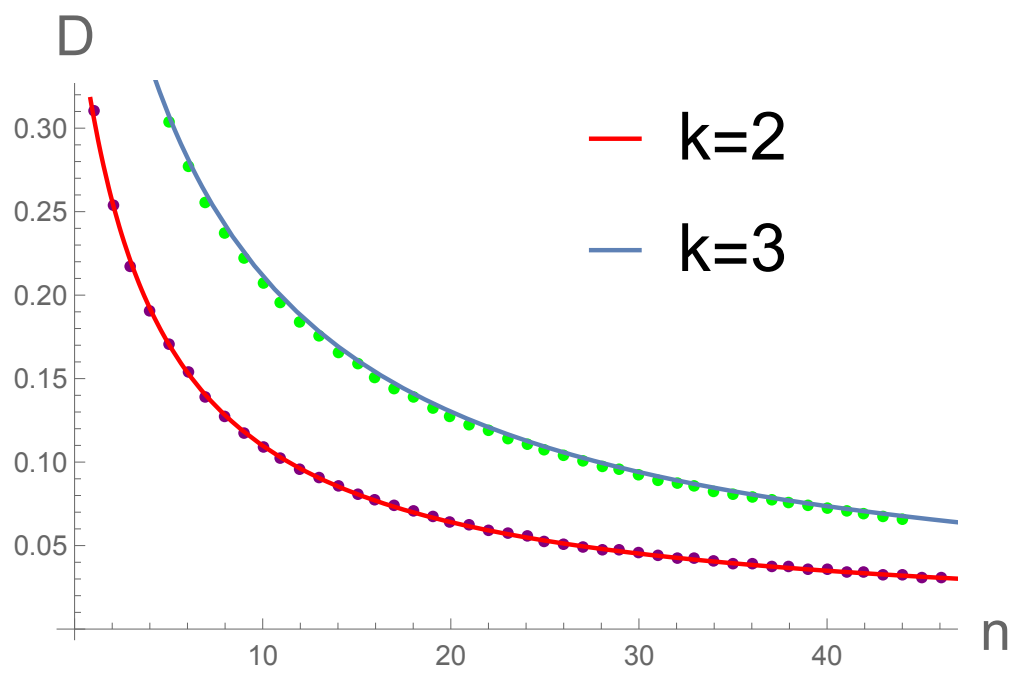

Figure 4. Displaying the left-hand side of eq. (4.17) for $k=2$ and $k=3$ for $W_{n}$ as a function of $n$, as well as the best fit curves of the form $D=\frac{B}{n}$. For $k=2$, we found $B \approx 1.537$, and for $k=3$, we found $B \approx 3.385$.

\section{Future direction: new dictionary entries}

In this section, we use intuition from bit threads [14] and from the fact that for states with a holographic dual, $E_{p}=E_{p}^{\infty}=E_{L O q}[13]$ to strengthen the $E_{W}=E_{p}$ conjecture. For a definition of these quantities see [15]. For our purposes, we will mainly use the fact that $E_{L O q}\left(\rho_{A B}\right)$ is roughly equal to the number of EPR pairs needed to get to $\rho_{A B}$ by means of only local operations.

Let $A$ and $B$ be boundary regions, and let the state on it be given by the density matrix $\rho_{A B}$. Consider the maximum flow $\Phi$ from $A$ and into $B .^{7}$ We interpret these as bit threads connecting EPR pairs living on the boundary as follows and imagine the flow lines that leave $A$ and end on $B$ correspond to EPR pairs between $A$ and $B$. If a thread does not end on $B$, then it should correspond to an EPR pair between $A$ and the purifying system $A^{\prime} B^{\prime}$. Likewise, the flow lines ending on $B$ and not coming from $A$ would be EPR pairs between $B$ and $A^{\prime} B^{\prime}$. We assume that the global boundary state is pure. ${ }^{8}$ This idea can be made more rigorous as follows:

Conjecture. Let $A$ and $B$ be disjoint boundary regions at a time symmetric slice $\Sigma, r_{A B}$ be their entanglement wedge restricted to $\Sigma$, and $\Phi$ be a maximizing flow from $A$ into $B$. Any (non-minimal) surface $\Gamma \subset \Sigma$ that partitions $r_{A B}$ into a region homologous to $A$ and a region homologous to $B$ is dual to a (suboptimal) purification $A^{\prime} B^{\prime}$ such that $A^{\prime} \subset \tilde{A}$ and $B^{\prime} \subset \tilde{B}$, where $\tilde{A}(\tilde{B})$ is the boundary region which is either connected to $A(B)$ by the flow lines of $\Phi$ without crossing $\Gamma$ or are connected to $B$ (A) while crossing $\Gamma$, and $\Phi(\Gamma)=S\left(A A^{\prime}\right)$.

\footnotetext{
${ }^{7}$ We can do this by simultaneously maximizing the flow out of both $A$ and $B^{c}$, which contains $A$, something permitted by the nesting property of bit threads.

${ }^{8}$ This can always be achieved via multiboundary wormhole completion.
} 
Note that the subsystems $A^{\prime} \subset \tilde{A}$ and $B^{\prime} \subset \tilde{B}$ need not be geometric. Moreover, since $A \tilde{A} B \tilde{B}$ is pure, a purification $A A^{\prime} B B^{\prime}$ always exists.

Intuitively, the conjecture gives EPR pairs from which the original $\rho_{A B}$ can be reached by local operations on $A$ and on $B$. Moreover, we may speculate that if $\Gamma$ is perpendicular to $\Phi$, then $A^{\prime}$ and $B^{\prime}$ are, respectively, the subsets of $\tilde{A}$ and of $\tilde{B}$ that minimize $S\left(A A^{\prime}\right)$ constrained to $A A^{\prime} B B^{\prime}$ being pure. It would be interesting to have a concrete prescription for selecting $A^{\prime} \subset \tilde{A}$ and $B \subset \tilde{B}$ that works for arbitrary ("wiggly") cuts.

If this conjecture holds, then it is clear then that $S\left(A A^{\prime}\right)$ is minimized when $\Gamma$ is the entanglement wedge cross-section. Combining our conjecture with max-flow min-cut implies the $E_{W}=E_{p}$ conjecture, ${ }^{9}$ as the area of the minimal cross-section of the entanglement wedge is given by the number of bit threads crossing it in this construction, due to its nature as a bottleneck for the flow from $A$ to $B$. Thus, it is also clear that for any cut $\Gamma$ there exist an $A^{\prime}$ and $B^{\prime}$ such that $\Phi(\Gamma)=S\left(A A^{\prime}\right)$.

Still, the conjecture places nontrivial constraints on the dimensionality of the purifying system. This is because the sum of the number of bit threads emerging from the $A$ system when maximizing the flow through $A$ and those emerging from the $B$ system when maximizing the flow through $B$ upper bounds the log of the dimensionality of the $A^{\prime} B^{\prime}$ system. Thus, we get

$$
\log d_{A^{\prime} B^{\prime}} \leq S(A)+S(B) .
$$

Note that this is much tighter than the upper bound given in [15]; this is not all that surprising, however, given that holographic states have much less entanglement than the generic quantum states considered in [15]. Moreover the overall holographic state is pure, and thus one does not get confounding entanglement of purification from considering classical mixtures.

It would be interesting to study the plausibility of this conjecture in toy models of holography, in particular suitable generalizations of the qutrit code [23], perfect tensors [24], or the random tensor model [25]. In these models one would be able to falsify our conjecture by finding a system $A B$ for which there is no optimal purification $A^{\prime} B^{\prime}$ with dimension $d_{A^{\prime} B^{\prime}}$ satisfying eq. (5.1). In these simpler models, it may also be possible to explicitly reconstruct $A^{\prime}$ and $B^{\prime}$ from given known $A$ and $B$, say by explicitly searching for the unitary that would extract either the $A^{\prime}$ or $B^{\prime}$ systems tensored with unentangled ancilla qubits from the systems in which they are conjectured to be contained; because the search space is much smaller in finite dimensional systems, this search is in principle feasible here.

Other interesting directions of future research include to either prove as disprove eq. (4.12) as an inequality valid for all quantum systems, to extend the results of the present paper to the fully covariant case, as well as connections between the results here and the Markov property of the vacuum [26].

\section{Conclusion}

In this paper, we have considered upper and lower bounds for several information theoretic quantities, including bounds on the conditional mutual information, tripartite information,

\footnotetext{
${ }^{9}$ In turn, the $E_{W}=E_{p}$ conjecture implies Ryu-Takayanagi as a special case.
} 


\begin{tabular}{|c|c|c|}
\hline & Lower Bound & Upper Bound \\
\hline $\begin{array}{l}\text { Mutual Information } \\
\text { I(A:B) }\end{array}$ & 0 & $2 \mathbf{E}_{\mathbf{p}}(\mathrm{A}: \mathrm{B})$ \\
\hline $\begin{array}{c}\text { Conditional Mutual Information } \\
\qquad \mathrm{I}(\mathrm{A}: \mathrm{B}-\mathrm{C})\end{array}$ & 0 & ${ }_{2} E_{p}^{G}(A C: B C)$ \\
\hline $\begin{array}{l}\text { Tripartite Information } \\
\text { I(A:B:C) }\end{array}$ & $\begin{array}{c}0, \text { and } \\
-2 E_{p}(A B: C)-2 E_{p}(B: C)\end{array}$ & $\begin{aligned} E_{p}^{G}(A C & : B C)+E_{p}^{G}(A B: B C) \\
& +E_{p}^{G}(A B: A C)\end{aligned}$ \\
\hline $\begin{array}{l}\text { Cyclic Information } \\
C_{k}\left(A_{1}, \ldots, A_{2 k+1}\right)\end{array}$ & 0 & $\sum_{i=1}^{n} E_{p}^{G}\left(A_{i}, \ldots, A_{i+k}: A_{i+k}, \ldots A_{i+n-1}\right)$ \\
\hline
\end{tabular}

Table 1. The main results are listed in the table. In standard black font are the results that only hold for holographic states. All others hold for general quantum states. Results that, to the best of the authors knowledge, are new are in red bold italics text (the upper bound on conditional mutual information was proved for $E_{W}$ in the language of bit threads in [14]). In blue bold text are the inequalities that were already known to hold for all quantum states. In addition to these results, we have also shown the holographic inequality in eq. (3.20), which does not fit neatly into this table. The general version of this inequality, pending the $E_{W}=E_{p}$ conjecture, is given by eq. (4.12). We have shown this to hold for several non-holographic states, but its general validity is still an open question.

and cyclic information. Despite being motivated by holography, we have shown these to hold for all quantum states. We have also found a new family of holographic inequalities for $E_{W}$, and provided evidence that the corresponding inequality for $E_{p}$ may be true for all quantum states. These results are summarized in table 1.

Finally, we conjectured a potential extension of the $E_{W}=E_{p}$ conjecture of $[12,13]$, which asserts that all cuts of the entanglement wedge are dual to purifications. If true, it may be possible to write such a map explicitly, which could lead to an efficient way of computing entanglement of purification.

\section{Acknowledgments}

We would like to thank Raphael Bousso, Veronika Hubeny, Adam Levine, Rob Myers, Daniel Parker, David Wakeham, Aron Wall, and Sean Weinberg for discussions. We also thank an anonymous referee for helpful comments, in particular with regards to sharpening the conjecture in section 5. This work is supported in part by the Berkeley Center for Theoretical Physics. N.B. is supported by the National Science Foundation, under grant number 82248-13067-44-PHPXH, and I.F.H. is supported by National Science Foundation under grant PHY-1521446.

Open Access. This article is distributed under the terms of the Creative Commons Attribution License (CC-BY 4.0), which permits any use, distribution and reproduction in any medium, provided the original author(s) and source are credited. 


\section{References}

[1] J.M. Maldacena, The large-N limit of superconformal field theories and supergravity, Int. J. Theor. Phys. 38 (1999) 1113 [Adv. Theor. Math. Phys. 2 (1998) 231] [hep-th/9711200] [INSPIRE].

[2] E. Witten, Anti-de Sitter space and holography, Adv. Theor. Math. Phys. 2 (1998) 253 [hep-th/9802150] [INSPIRE].

[3] S. Ryu and T. Takayanagi, Aspects of holographic entanglement entropy, JHEP 08 (2006) 045 [hep-th/0605073] [INSPIRE].

[4] S. Ryu and T. Takayanagi, Holographic derivation of entanglement entropy from AdS/CFT, Phys. Rev. Lett. 96 (2006) 181602 [hep-th/0603001] [InSPIRE].

[5] V.E. Hubeny, M. Rangamani and T. Takayanagi, A covariant holographic entanglement entropy proposal, JHEP 07 (2007) 062 [arXiv:0705.0016] [INSPIRE].

[6] N. Bao et al., The holographic entropy cone, JHEP 09 (2015) 130 [arXiv:1505.07839] [INSPIRE].

[7] P. Hayden, M. Headrick and A. Maloney, Holographic mutual information is monogamous, Phys. Rev. D 87 (2013) 046003 [arXiv: 1107.2940] [INSPIRE].

[8] M. Van Raamsdonk, Building up spacetime with quantum entanglement, Gen. Rel. Grav. 42 (2010) 2323 [arXiv: 1005.3035] [INSPIRE].

[9] J. Maldacena and L. Susskind, Cool horizons for entangled black holes, Fortsch. Phys. 61 (2013) 781 [arXiv:1306.0533] [INSPIRE].

[10] T. Faulkner et al., Gravitation from entanglement in holographic CFTs, JHEP 03 (2014) 051 [arXiv: 1312.7856] [INSPIRE].

[11] B. Swingle and M. Van Raamsdonk, Universality of gravity from entanglement, arXiv: 1405.2933 [INSPIRE].

[12] P. Nguyen et al., Entanglement of purification: from spin chains to holography, JHEP 01 (2018) 098 [arXiv: 1709.07424] [INSPIRE].

[13] T. Takayanagi and K. Umemoto, Holographic entanglement of purification, arXiv: 1708.09393 [INSPIRE].

[14] M. Headrick, V.E. Hubeny, A. Lawrence and M. Rangamani, Causality \& holographic entanglement entropy, JHEP 12 (2014) 162 [arXiv:1408.6300] [INSPIRE].

[15] B.M. Terhal et al., The entanglement of purification, J. Math. Phys. 43 (2002) 4286 [quant-ph/0202044].

[16] S. Bagchi and A.K. Pati,Monogamy, polygamy, and other properties of entanglement of purification, Phys. Rev. A 91 (2015) 042323 [arXiv:1502.01272].

[17] M. Headrick and T. Takayanagi, A holographic proof of the strong subadditivity of entanglement entropy, Phys. Rev. D 76 (2007) 106013 [arXiv:0704.3719] [INSPIRE].

[18] A.C. Wall, Maximin surfaces and the strong subadditivity of the covariant holographic entanglement entropy, Class. Quant. Grav. 31 (2014) 225007 [arXiv:1211.3494] [INSPIRE].

[19] C. Akers, J. Koeller, S. Leichenauer and A. Levine, Geometric constraints from subregion duality beyond the classical regime, arXiv:1610.08968 [INSPIRE]. 
[20] M. Freedman and M. Headrick, Bit threads and holographic entanglement, Commun. Math. Phys. 352 (2017) 407 [arXiv: 1604.00354] [INSPIRE].

[21] E. Wakakuwa, Operational resource theory of non-markovianity, arXiv:1709.07248.

[22] M. Rangamani and M. Rota, Entanglement structures in qubit systems, J. Phys. A 48 (2015) 385301 [arXiv:1505.03696] [INSPIRE].

[23] A. Almheiri, X. Dong and D. Harlow, Bulk locality and quantum error correction in AdS/CFT, JHEP 04 (2015) 163 [arXiv:1411.7041] [INSPIRE].

[24] F. Pastawski, B. Yoshida, D. Harlow and J. Preskill, Holographic quantum error-correcting codes: toy models for the bulk/boundary correspondence, JHEP 06 (2015) 149 [arXiv: 1503.06237] [INSPIRE].

[25] P. Hayden et al., Holographic duality from random tensor networks, JHEP 11 (2016) 009 [arXiv: 1601.01694] [INSPIRE].

[26] H. Casini, E. Testé and G. Torroba, Markov property of the conformal field theory vacuum and the a-theorem, Phys. Rev. Lett. 118 (2017) 261602 [arXiv:1704.01870] [INSPIRE]. 